\title{
A NOTE ON GENERALIZED LICHNEROWICZ-OBATA THEOREMS FOR RIEMANNIAN FOLIATIONS
}

\author{
Hong Kyung Pak and Jeong Hyeong Park
}

\begin{abstract}
It was obtained in [5] generalized Lichnerowicz and Obata theorems for Riemannian foliations, which reduce to the results on Riemannian manifolds for the point foliations. Recently in [3], they studied a generalized Obata theorem for Riemannian foliations admitting transversal conformal fields. Each transversal conformal field is a $\lambda$-automorphism with $\lambda=1-\frac{2}{q}$ in the sense of [8]. In the present paper, we extend certain results established in [3] and study Riemannian foliations admitting $\lambda$-automorphisms with $\lambda \geq 1-\frac{2}{q}$.
\end{abstract}

\section{Introduction}

The study of relationships between the eigenvalues of the Laplacian and geometric quantities on a Riemannian manifold is one of interesting topics in Riemannian geometry. Among all the eigenvalues, the smallest positive eigenvalue plays the most important role. For example, the standard Lichnerowicz comparison theorem ([6]) says that if the Ricci operator $\rho$ of a closed connected Riemannian manifold $M$ of dimension $m \geq 2$ satisfies $\rho \geq c(m-1)$ id for some $c>0$, where id denotes the identity operator, then the smallest positive eigenvalue $\alpha$ of the Laplacian $\Delta$ acting on functions satisfies $\alpha \geq \mathrm{cm}$. The Obata theorem ([7]) says that equality occurs if and only if the $M$ is isometric to the standard $m$-sphere with constant sectional curvature $c$. In particular, when $M$ is Einstein with constant scalar curvature $\sigma$, the four following conditions are equivalent to each other.

(C1) $M$ is isometric to the standard $m$-sphere with radius $\frac{1}{\sqrt{c}}$ in the $(m+1)$ dimensional Euclidean space,

(C2) $M$ admits a non-homothetic conformal vector field,

Received December 10, 2009; Revised January 17, 2011.

2010 Mathematics Subject Classification. Primary 53C20; Secondary 57R30.

Key words and phrases. Riemannian foliation, generalized Lichnerowicz-Obata theorem, $\lambda$-automorphism, transversally Einstein foliation.

Research of H. K. Pak and J. H. Park was supported by Basic Science Research Program through the National Research Foundation of Korea(NRF) funded by the Ministry of Education, Science and Technology(2009-0087201). 
(C3) $M$ admits a non constant function $f$ satisfying $\nabla \nabla f+c f g=0$,

(C4) $M$ admits a non constant function $f$ satisfying $\Delta f=c m f$.

Here $\nabla$ denotes the Levi-Civita connection of $g$. Lee and Richardson ([5]) generalized these results to the context of Riemannian foliations. That is,

Theorem 1.1. Let $(M, g, \mathcal{F})$ be a closed connected Riemannian manifold with Riemannian foliation $\mathcal{F}$ of codimension $q \geq 2$. If the transversal Ricci operator $\rho_{D}$ of satisfies $\rho_{D} \geq c(q-1)$ id for some $c>0$, then the smallest positive eigenvalue $\alpha_{B}$ of the basic Laplacian $\Delta_{B}$ acting on basic functions satisfies $\alpha_{B} \geq c q$. In addition, the equality occurs if and only if

(1) the leaf space $M / \mathcal{F}$ is isometric to the space of orbits of a discrete subgroup of $O(q)$ acting on the standard q-sphere with constant sectional curvature $c$,

(2) if we choose the metric on $M$ so that the mean curvature form $\kappa$ is basic, then $\mathcal{F}$ is harmonic,

(3) each level set of the $\alpha_{B}$ eigenfunction is the set of the leaves corresponding to a latitude of the q-sphere, and the volume of this level set is the volume of the maximum leaf times the volume of the latitude.

Theorem 1.1 provides a relationship between the above conditions (C1) and (C4). Related to the condition (C2), Jung and Jung ([3]) recently obtained a generalized Obata theorem (Theorem 5.9) for Riemannian foliations admitting transversal conformal fields.

Theorem 1.2. Let $(M, g, \mathcal{F})$ be as in Theorem 1.1. Let $\kappa$ be basic such that $\delta_{B} \kappa=0$ and the transversal scalar curvature $\sigma_{D}$ be non-zero constant. Assume that $\rho_{D} \geq \frac{\sigma_{D}}{q}$ id. If $M$ admits a transversal non-Killing conformal field, then $\mathcal{F}$ is transversally isometric to the action of a finite subgroup of $O(q)$ acting on the standard $q$-sphere with constant sectional curvature $c=\frac{\sigma_{D}}{q(q-1)}$.

Geometric transversal infinitesimal automorphisms such as transversal Killing, affine, projective, conformal, Jacobi fields have been attacked by many peoples. These are all examples of $\lambda$-automorphisms in the sense of [8]. For instance, each transversal Killing field is a $\lambda$-automorphism for all $\lambda \in \mathbb{R}$. Each transversal conformal and projective field is a $\left(1-\frac{2}{q}\right)$-automorphism and $\left(-\frac{2}{q+1}\right)$-automorphism respectively.

In the present paper we discuss a Riemannian foliation admitting a transversal conformal field from the viewpoint of $\lambda$-automorphisms. Then we improve the proof of Theorem 1.2 given in [3] by showing that the foliation is harmonic. When the foliation is transversally Einstein, we prove that a non-homothetic conformal field appeared in (C2) can be replaced by a $\lambda$-automorphism with $\lambda \geq 1-\frac{2}{q}$. Moreover, we generalize the previous Obata's result concerning the conditions $(\mathrm{C} 1) \sim(\mathrm{C} 4)$ to transversally Einstein foliations.

The authors would like to thank the referee for his valuable suggestions and comments. 


\section{Preliminaries}

Let $(M, g, \mathcal{F})$ be a $m$-dimensional closed connected Riemannian manifold with Riemannian foliation $\mathcal{F}$ of codimension $q:=m-p \geq 2$ and a bundle-like metric $g$. It is given by an exact sequence of vector bundles

$$
0 \rightarrow \mathcal{V} \rightarrow T M \stackrel{\pi}{\rightarrow} Q \rightarrow 0,
$$

where $\mathcal{V}$ is the tangent bundle and $Q$ the normal bundle of $\mathcal{F}$. The metric $g$ determines an orthogonal decomposition $T M=\mathcal{V} \oplus \mathcal{H}$. We identify $\mathcal{H}$ with $Q$ by an isometric splitting

$$
\left(Q, g_{Q}\right) \cong\left(\mathcal{H}, g_{\mathcal{H}}\right)
$$

We have an associated exact sequence of Lie algebras

$$
0 \rightarrow \Gamma(\mathcal{V}) \rightarrow V(\mathcal{F}) \stackrel{\pi}{\rightarrow} \bar{V}(\mathcal{F}) \rightarrow 0,
$$

where $V(\mathcal{F}):=\{Y \in \Gamma(T M) \mid[V, Y] \in \Gamma(\mathcal{V})$ for all $V \in \Gamma(\mathcal{V})\}$ and $\bar{V}(\mathcal{F}):=$ $\{s \in \Gamma(Q) \mid s=\pi(Y), Y \in V(\mathcal{F})\}$, called the space of transversal infinitesimal automorphisms of $\mathcal{F}$. Here and hereafter, we denote by $\Gamma(\cdot)$ the space of all smooth sections of a vector bundle $(\cdot)$. The transversal Levi-Civita connection $D$ on $Q$ is a torsion free and metric connection with respect to $g_{Q}$ (see [9], $(5.9))$.

Throughout this paper, we use the following notations:

$\tau$ : the tension field of $\mathcal{F}$,

$\operatorname{grad}_{D} f$ : the transversal gradient of a function $f \in C^{\infty}(M)$,

$\operatorname{div}_{D} s$ : the transversal divergence of $s \in \Gamma(Q)$,

$\rho_{D}$ : the transversal Ricci operator,

$\sigma_{D}$ : the transversal scalar curvature,

$\Delta$ : the Laplacian acting on $\Gamma(Q)$,

$\theta(Y)$ : the transversal Lie derivative operator for $Y \in V(\mathcal{F})$,

$A_{D}(Y):=\theta(Y)-D_{Y}$ for $Y \in V(\mathcal{F})$.

In what follows, we assume that $\tau \in \bar{V}(\mathcal{F})$. This assumption is based on the tenseness theorem ([1]) and a result ([5], Corollary 4.2).

The basic complex $\left(\Omega_{B}, d_{B}:=\left.d\right|_{\Omega_{B}}\right)$ is a subcomplex of the de Rham complex $(\Omega(M), d)$, where

$$
\Omega_{B}:=\left\{\omega \in \Omega(M) \mid i_{V} \omega=\theta(V) \omega=0 \text { for all } V \in \Gamma(\mathcal{V})\right\} .
$$

Its cohomology

$$
H_{B}:=H\left(\Omega_{B}, d_{B}\right)
$$

is called the basic cohomology of $\mathcal{F}$. We often use the following identification by means of (2.1) and $g_{Q}$-duality

$$
\bar{V}(\mathcal{F}) \cong \Omega_{B}^{1}
$$

For convenience $\mathcal{F}$ is assumed to be oriented and transversally oriented. The transversal volume form associated to $\mathcal{F}$ is an element $\nu \in \Omega_{B}^{q}$ with $d \nu=0$. The characteristic form is the $p$-form $\chi_{\mathcal{F}}:=* \nu$, expressed in terms of the Hodge 
star operator $*$. The Riemannian volume form $\mu \in \Omega^{m}(M)$ is then given by $\mu=\nu \wedge \chi_{\mathcal{F}}$.

We use the same notation $g_{Q}$ for the local scalar product on tensors of $Q$ and $|\cdot|^{2}:=g_{Q}(\cdot, \cdot)$. Let $\langle$,$\rangle be the global scalar product defined by$

$$
\langle\phi, \psi\rangle:=\int_{M} g_{Q}(\phi, \psi) \mu .
$$

It is well-known that on $\Omega_{B}$

$$
\langle\phi, \psi\rangle=\int_{M} \phi \wedge \bar{*} \psi \wedge \chi_{\mathcal{F}},
$$

where $\bar{*}$ is the star operator associated to the holonomy-invariant metric $g_{Q}$. Then the formal adjoint $\delta_{B}$ of $d_{B}$ on $\Omega_{B}^{r}$ with respect to $\langle$,$\rangle is given by the$ formula

$$
\delta_{B}=(-1)^{r(q+1)+1} \bar{*} d_{T} \bar{*}=\delta_{T}+i_{\tau},
$$

where

$$
d_{T}:=d_{B}-\kappa \wedge, \quad \delta_{T}:=(-1)^{r(q+1)+1} \bar{*} d_{B} \bar{*} .
$$

Here $\kappa$ is the mean curvature 1 -form of $\mathcal{F}$ which is the $g_{Q}$-dual to $\tau$. It should be noted that $d_{T}$ and $\delta_{T}$ are formal adjoint with respect to $\langle$,$\rangle . The basic$ Laplacian acting on $\Omega_{B}$ is defined by

$$
\Delta_{B}:=d_{B} \delta_{B}+\delta_{B} d_{B}
$$

\section{Main results}

For our purpose we start with recalling the notion of $\lambda$-automorphisms discussed in [8]. $s \in \bar{V}(\mathcal{F})$ is called a $\lambda$-automorphism for $\lambda \in \mathbb{R}$ if it satisfies

$$
\Delta s-D_{\tau} s-\rho_{D}(s)-\lambda \operatorname{grad}_{D} \operatorname{div}_{D} s=0,
$$

or equivalently the $g_{Q}$-dual $\omega$ satisfies

$$
-\operatorname{tr} D^{2} \omega-\rho_{D}(\omega)+\lambda d_{B} \delta_{T} \omega=0 .
$$

As examples, if $s$ is a transversal conformal field, that is,

$$
\theta(s) g_{Q}=2 f_{s} g_{Q},
$$

then

$$
\operatorname{div}_{D} s=q f_{s}, \quad \Delta s=D_{\tau} s+\rho_{D}(s)+\left(1-\frac{2}{q}\right) \operatorname{grad}_{D} \operatorname{div}_{D} s .
$$

In particular, if $f_{s}$ is constant, $s$ is called a transversal homothetic field. A transversal conformal field is a $\left(1-\frac{2}{q}\right)$-automorphism. The converse is not true unless $\mathcal{F}$ is harmonic $(\kappa=0)([8])$. When we refer in the sequel to a transversal conformal field $s$, we always mean by $f_{s}$ the function appearing in (3.3). 
Lemma 3.1. Let $(M, g, \mathcal{F})$ be a closed connected Riemannian manifold with Riemannian foliation $\mathcal{F}$ of codimension $q \geq 2$. Suppose that $\kappa$ is basic and the transversal scalar curvature $\sigma_{D}$ is constant. If $M$ admits a transversal conformal field $s$, then it holds

$$
\Delta_{B} f_{s}=\frac{\sigma_{D}}{q-1} f_{s}+\tau\left(f_{s}\right) .
$$

Proof. Let $s$ be a $\lambda$-automorphism and $\omega$ be its $g_{Q}$-dual. From the Weitzenböck formula

$$
d_{B} \delta_{T} \omega+\delta_{T} d_{B} \omega=-\operatorname{tr} D^{2} \omega+\rho_{D}(\omega),
$$

we see that (3.2) is equivalent to the formula

$$
(1+\lambda) d_{B} \delta_{T} \omega+\delta_{T} d_{B} \omega=2 \rho_{D}(\omega) .
$$

Since $\sigma_{D}$ is constant, a transversal conformal field $s$ satisfies

$$
\delta_{T}\left(\rho_{D}(\omega)\right)=-g_{Q}\left(S_{D}, D \omega\right)=-\sigma_{D} f_{s},
$$

where $S_{D}$ denotes the transversal Ricci tensor. It follows from (3.4) and (3.8) that

$$
(q-1) \delta_{T} d_{B} f_{s}=\sigma_{D} f_{s}
$$

which follows (3.7) by means of (2.5).

It is noted that (3.7) was derived by another way via the formula ([3], Proposition 5.5)

$$
\theta(s) \sigma_{D}=2(q-1) \delta_{T} d_{B} f_{s}-2 \sigma_{D} f_{s} .
$$

Corollary 3.2. Let $(M, g, \mathcal{F}), \kappa$ and $\sigma_{D}$ be as in Lemma 3.1. Further suppose that $\delta_{B} \kappa=0$. If $M$ admits a transversal non-homothetic conformal field $s$, then $\sigma_{D}$ is positive.

Proof. Observe from $\delta_{B} \kappa=0$ that

$$
\left\langle\tau\left(f_{s}\right), f_{s}\right\rangle=\frac{1}{2}\left\langle d_{B}\left(f_{s}^{2}\right), \kappa\right\rangle=0 .
$$

Then Lemma 3.1 implies

$$
\left\|d_{B} f_{s}\right\|^{2}=\left\langle\frac{\sigma_{D}}{q-1} f_{s}+\tau\left(f_{s}\right), f_{s}\right\rangle=\frac{\sigma_{D}}{q-1}\left\|f_{s}\right\|^{2}
$$

so that $\sigma_{D}>0$.

It is well-known the transversal Hodge isomorphism

$$
H_{B}^{r} \simeq \mathcal{H}_{B}^{r}
$$

where $\mathcal{H}_{B}^{r}:=\operatorname{ker} \Delta_{B} \cap \Omega_{B}^{r}$ and the tautness theorem $\left([\kappa]=0\right.$ in $\left.H_{B}^{1}\right)$ under the positivity of the transversal Ricci curvature (see [12], (7.51) and (8.22)). In the same situation we may show a more stronger result, the harmonicity theorem $(\kappa=0)$. Here we give a direct simple proof by using the transversal Weitzenböck formula for $\phi \in \Omega_{B}^{r}$

$$
\Delta_{B} \phi=D_{\mathrm{tr}}^{*} D_{\mathrm{tr}} \phi+F(\phi)+A_{\tau} \phi,
$$


where $D_{\mathrm{tr}}^{*} D_{\mathrm{tr}}$ is a non-negative and formally self-adjoint operator ([3]).

Lemma 3.3. Let $(M, g, \mathcal{F})$ be as in Lemma 3.1. Suppose that $\kappa$ is basic such that $\delta_{B} \kappa=0$. If $\rho_{D}>0$, then $\mathcal{F}$ is harmonic.

Proof. Since $M$ is closed, $\kappa \in \Omega_{B}^{1}$ implies $d \kappa=0$ (see [12], (7.5)). Thus $\kappa \in \mathcal{H}_{B}^{1}$. Note that

$$
\left(A_{\tau} \kappa\right)(t)=-\kappa\left(A_{\tau} t\right)=\kappa\left(D_{t} \tau\right)=\frac{1}{2} d|\kappa|^{2}(t)
$$

for any $t \in \bar{V}(\mathcal{F})$. Then (3.9) combined with (3.10) yields

$$
D_{\mathrm{tr}}^{*} D_{\mathrm{tr}} \kappa+\rho_{D}(\kappa)+\frac{1}{2} d|\kappa|^{2}=0 .
$$

It follows that

$$
\begin{aligned}
0 & =\left\langle D_{\mathrm{tr}}^{*} D_{\operatorname{tr}} \kappa+\rho_{D}(\kappa)+\frac{1}{2} d|\kappa|^{2}, \kappa\right\rangle \\
& =\left\|D_{\operatorname{tr}} \kappa\right\|^{2}+\left\langle\rho_{D}(\kappa), \kappa\right\rangle .
\end{aligned}
$$

Therefore the Bochner technique shows $\kappa=0$.

Remark. Lemma 3.3 is proved in [2] in a somewhat different way. Its proof is based on the exactness property of $\kappa$ from the positivity condition of $\rho_{D}$.

Now we are in a position to improve Theorem 1.2 in light of Lemma 3.3. It should be noted that the tautness theorem used in the proof of [3] can be replaced by the harmonicity theorem in our proof.

Theorem 3.4. Let $(M, g, \mathcal{F})$ be as in Lemma 3.1. Suppose that $\kappa$ is basic such that $\delta_{B} \kappa=0$ and $\sigma_{D}$ is constant. Assume that $\rho_{D} \geq \frac{\sigma_{D}}{q} \mathrm{id}$. If $M$ admits a transversal non-homothetic conformal field $s$, then $\mathcal{F}$ is harmonic and transversally isometric to the action of a finite subgroup of $O(q)$ acting on the standard $q$-sphere with constant sectional curvature $c=\frac{\sigma_{D}}{q(q-1)}$.

Proof. Since $\sigma_{D}$ is constant, Corollary 3.2 yields $\sigma_{D}>0$, and so $\rho_{D}>0$. Then $\mathcal{F}$ is harmonic by Lemma 3.3 .

In addition, we get from Lemma 3.1

$$
\Delta_{B} f_{s}=\frac{\sigma_{D}}{q-1} f_{s}
$$

If we take $c:=\frac{\sigma_{D}}{q(q-1)}$, then $\alpha_{B} \leq c q$ by means of (3.11). Hence Generalized Lichnerowicz Theorem (the first part of Theorem 1.1) deduces that $\alpha_{B}=c q$. Therefore the proof is completed by Generalized Obata Theorem (the second part of Theorem 1.1).

Remarks. (1) Observe that (3.11) means $f_{s}=-\frac{1}{q} \delta_{T} \omega$ is an eigenfunction with the eigenvalue $\alpha_{B}$. Thus each level set of $f_{s}$ is the set of the leaves corresponding to a latitude of the $q$-sphere, and the volume of this level set is the volume of the maximum leaf times the volume of the latitude. 
(2) It was proved in [9] that assuming $\sigma_{D} \leq 0$ if $s \in \bar{V}(\mathcal{F})$ is a transversal conformal field which satisfies $\left\langle B_{D}^{0}(s) s, \tau\right\rangle \geq 0$, where

$$
B_{D}^{\lambda}(s):=A_{D}(s)+{ }^{t} A_{D}(s)+\lambda\left(\operatorname{div}_{D} s\right) \mathrm{id},
$$

then $s$ is a transversal Killing field.

(3) In [10] the author considered a notion of foliated transformations for manifolds with Riemannian foliations. Each foliated conformal (resp. foliated isometric) transformation is defined by a transformation preserving the leaves such that the induced map on their normal bundles is conformal (resp. isometric). It has been studied the conditions when a foliated conformal transformation is to be foliated isometric in terms of transversal Ricci or scalar curvatures.

(4) If we further add the assumption that $f_{s}$ is an eigenfunction of $\Delta_{B}$ with some eigenvalue $\tilde{\alpha_{B}}$ in Theorem 3.4, the harmonicity of $\mathcal{F}$ may be proved by another way. Indeed, Lemma 3.1 in this case becomes

$$
\tau\left(f_{s}\right)=\left(\tilde{\alpha_{B}}-\frac{\sigma_{D}}{q-1}\right) f_{s} \geq\left(\alpha_{B}-c q\right) f_{s},
$$

so that

$$
0 \geq\left(\alpha_{B}-c q\right)\left\|f_{s}\right\|^{2}
$$

Thus Generalized Lichnerowicz Theorem implies $\alpha_{B}=c q$. It follows from Generalized Obata theorem that $\mathcal{F}$ is harmonic.

Related to the Obata's result stated in Section 1, we now consider the particular case where $\mathcal{F}$ is transversally Einstein, i.e., $\rho_{D}=\frac{\sigma_{D}}{q}$ id. Note that any $\lambda$-automorphism $s$ satisfies (3.8). In this situation, applying $\delta_{T}$ and using $\sigma_{D}$ constant give rise to

$$
(1+\lambda) \delta_{T} d_{B} f_{s}=2 \frac{\sigma_{D}}{q} f_{s}
$$

Therefore by a similar argument as in Theorem 3.4 we conclude:

Theorem 3.5. Let $(M, g, \mathcal{F})$ and $\kappa$ be as in Theorem 3.4. Further assume that $\mathcal{F}$ is transversally Einstein. If $M$ admits a $\lambda$-automorphism $s$ with $\lambda \geq$ $1-\frac{2}{q}$ whose transversal divergence is not constant, then $\mathcal{F}$ is harmonic and transversally isometric to the action of a finite subgroup of $O(q)$ acting on the standard $q$-sphere with constant sectional curvature $c=\frac{2 \sigma_{D}}{(1+\lambda) q^{2}}$.

Proof. (3.12) implies

$$
(1+\lambda) \delta_{T} d_{B} f=2 \frac{\sigma_{D}}{q} f
$$

where $f:=\delta_{T} \omega$. Then

$$
\Delta_{B} f=\frac{2 \sigma_{D}}{(1+\lambda) q} f+\tau(f) .
$$

Since $\operatorname{div}_{D} s$ is not constant and $\delta_{B} \kappa=0$, we get $\sigma_{D}>0$. Hence $\rho_{D}>0$. Thus $\mathcal{F}$ is harmonic by Lemma 3.3. Then (3.13) induces $\alpha_{B} \leq c q$. 
On the other hand, we find from $\lambda \geq 1-\frac{2}{q}$

$$
\rho_{D}=\frac{\sigma_{D}}{q} \mathrm{id} \geq c(q-1) \mathrm{id},
$$

which follows $\alpha_{B}=c q$. Therefore we complete the proof by virtue of Theorem 1.1 .

Remarks. (1) Theorem 3.5 shows that the constant sectional curvature attains the maximum $c=\frac{\sigma_{D}}{q(q-1)}$ at which $s$ is a transversal non-homothetic conformal field.

(2) It was obtained in [8] that a $\lambda$-automorphism $s$ whose transversal divergence is constant is transversal Killing if and only if $\left\langle B_{D}^{0}(s) s, \tau\right\rangle \geq 0$.

Corollary 3.6. Under the same assumptions as in Theorem 3.5, the four following statements are equivalent to each other.

(F1) $\mathcal{F}$ is harmonic and transversally isometric to the action of a finite subgroup of $O(q)$ acting on the standard $q$-sphere with constant sectional curvature $c$.

(F2) $M$ admits a transversal non-homothetic conformal field,

(F3) $M$ admits a non-constant basic function $f$ satisfying $D D f+c f g_{Q}=0$,

(F4) $M$ admits a non-constant basic function $f$ satisfying $\delta_{T} d_{B} f=c q f$,

where $c=\frac{\sigma_{D}}{q(q-1)}$.

Proof. Generalized Obata Theorem says $(\mathrm{F} 1) \Leftrightarrow(\mathrm{F} 4)$. Besides Theorem 3.4 means $(\mathrm{F} 2) \Rightarrow(\mathrm{F} 1)$. Furthermore, we easily see that (F3) $\Rightarrow$ (F2). Indeed, if we take $s=D f$, then (F3) implies $\theta(s) g_{Q}=2 D D f=-2 c f g_{Q}$, so $s$ is a desired one. Therefore it suffices to show $(\mathrm{F} 4) \Rightarrow(\mathrm{F} 3)$.

To begin with, we observe that by a similar argument of Theorem 3.4, (F4) yields $0<c=\frac{\sigma_{D}}{q(q-1)}$, and so $\mathcal{F}$ is harmonic. Hence

$$
c q f=\delta_{T} d_{B} f=\Delta_{B} f .
$$

Furthermore it holds for any $h \in \Omega_{B}^{0}$ that $([5])$

$$
\frac{1}{2} \Delta_{B}\left|d_{B} h\right|^{2}=g_{Q}\left(\Delta_{B} d_{B} h, d_{B} h\right)-\left|D d_{B} h\right|^{2}-S_{D}(D h, D h),
$$

where $S_{D}(s, t):=g_{Q}\left(\rho_{D}(s), t\right)$ for $s, t \in \bar{V}(\mathcal{F})$. Then (3.14) and (3.15) together with $h=f$ imply

$$
\begin{aligned}
0 & =\left\langle\rho_{D}(D f), D f\right\rangle-\left\|\Delta_{B} f\right\|^{2}+\|D D f\|^{2} \\
& =\frac{\sigma_{D}}{q}\|D f\|^{2}-\|c q f\|^{2}+c^{2} q\|f\|^{2}+\left\|D D f+c f g_{Q}\right\|^{2} \\
& =\left\|D D f+c f g_{Q}\right\|^{2},
\end{aligned}
$$

which follows (F3). 
Remarks. (1) We informed later that $(F 1) \Leftrightarrow(F 3)$ was proved in [4] in a more general setting.

(2) Corollary 3.6 is still true even if we replace the transversally Einstein condition by a weaker condition $S_{D} \geq c(q-1) g_{Q}$.

\section{References}

[1] D. Domínguez, Finiteness and tenseness theorems for Riemannian foliations, Amer. J. Math. 120 (1998), no. 6, 1237-1276.

[2] G. Habib, Eigenvalues of the basic Dirac operator on quaternion-Kahler foliations, Ann. Global Anal. Geom. 30 (2006), no. 3, 286-298.

[3] M. J. Jung and S. D. Jung, Riemannian foliations admitting transversal conformal fields, Geom. Dedicata 133 (2008), 155-168.

[4] S. D. Jung, K. R. Lee, and K. Richardson, Generalized Obata theorem and its applications on foliations, ArXiv:0908.4545v1 [math.DG], 2009.

[5] J. Lee and K. Richardson, Lichnerowicz and Obata theorems for foliations, Pacific J. Math. 206 (2002), no. 2, 339-357.

[6] A. Lichnerowicz, Géométrie des groupes de transformations, Travaux et Recherches Mathématiques III, Dunod, Paris, 1958.

[7] M. Obata, Certain conditions for a Riemannian manifold to be isometric with a sphere, J. Math. Soc. Japan 14 (1962), 333-340.

[8] H. K. Pak, $\lambda$-automorphisms of a Riemannian foliation, Ann. Global Anal. Geom. 13 (1995), no. 3, 281-288

[9] ㄴautomorphisms of a Riemannian foliation II, Geom. Dedicata 66 (1997), no. $1,19-25$.

[10] Geometry of conformal transformations of foliated Riemannian manifolds, Adv. Stud. Contemp. Math. 9 (2004), no. 1, 33-40.

[11] J. H. Park and S. Yorozu, Transverse fields preserving the transverse Ricci field of a foliation, J. Korean Math. Soc. 27 (1990), no. 2, 167-175.

[12] Ph. Tondeur, Geometry of Foliations, Monographs in Math., Birkhäuser, 1997.

HONG KYUNG PAK

Department of Mathematics

DAEGU HAANY UNIVERSITY

KYUNGSAN 712-715, KOREA

E-mail address: hkpak@dhu.ac.kr

JEONG HyEONG PARK

Department of Mathematics

SUNGKYUNKWAN UNIVERSITY

SuwOn 440-746, KoreA

E-mail address: parkj@skku.edu 